\title{
Validation of the Brazilian Children's Test of Pseudoword Repetition in Portuguese speakers aged 4 to 10 years
}

F.H. Santos and O.F.A. Bueno
Departamento de Psicobiologia, Escola Paulista de Medicina,

Universidade Federal de São Paulo, São Paulo, SP, Brasil,
Correspondence

O.F.A. Bueno

Rua Botucatu, 862, 1ำ andar

04023-062 São Paulo, SP

Brasil

Fax: +55-11-5572-5092

E-mail: ofabueno@psicobio.epm.br

Research supported by FAPESP (No. 99/11989-4), AFIP and CAPES.

Part of a PhD thesis presented by

F.H. Santos to the Departamento de

Psicologia, EPM, UNIFESP, São Paulo, SP, Brazil.

Some of these results were presented

at the IV Congresso Brasileiro de

Neuropsicologia (Rio de Janeiro,

RJ, Brazil, 1999), and the Symposium

"Bridging Basic Neuroscience and

Clinical Research to Benefit the

Society" (Rio de Janeiro, RJ, Brazil,

November 20-26, 2001).

Received September 18, 2002

Accepted August 14, 2003 $\ldots \ldots \ldots \ldots \ldots \ldots \ldots$ Introduction

Working memory is a system that holds and manipulates information for short periods of time. According to the model of Baddeley and Hitch (1), it comprises at least three components: the visuospatial sketchpad for visuospatial information, the phonological loop for verbal information, and the central executive, a limited-capacity circuit responsible for the control of attention and manipulation of information (1).
The phonological loop contains a phonological store to retain speech-based information and a rehearsal or articulatory loop that serves to maintain decaying representations in the phonological store (2). The phonological loop plays an important role in language skills in early years, for instance in learning to read, in comprehension of spoken language, and in vocabulary acquisition (3). The main function of this component is to provide temporary storage of the unfamiliar sound structures of new words from which 
more stable lexical representations are gradually constructed (4).

There is evidence that nonword (also called pseudoword) repetition, as well as conventional tests of verbal short-term memory such as Digit Span, are constrained by the phonological loop (2). A pseudoword consists of a stimulus within the structural rules of a natural language, i.e., it can be read, written and repeated but has no conceptual meaning or semantic value in the current lexicon of that language. Because the phonological form of a pseudoword is necessarily unfamiliar, subjects have to rely heavily upon the capacity of their phonological memory system to encode and keep the novel phonological sequence in a nondegraded form for subsequent articulatory output (4). However, nonword repetition skill is influenced by the extent to which the stimuli resemble words; repetition is easier for nonwords that have a phonological or morphological structure close to real words (5-7).

The Children's Test of Nonword Repetition (CNRep) requires transient storage of unfamiliar phonological forms (8). The child hears each pseudoword (for example, "loddernapish") and then attempts to repeat it immediately. The test consists of 40 pseudowords of different length (2, 3, 4 and 5 syllables), presented in the same order to all participants. The dependent measure is the number of correct responses.

For young children or children with learning disabilities, the CNRep task is more suitable than the auditory Digit Span, the classical measure of the phonological loop, because many children have difficulty in understanding the task. For instance, many children have difficulty in waiting for the sequence of numbers to be completed before they begin to repeat them, or tend to repeat the sequence in reverse order. For children, repeating a single word spoken by an adult seems a natural response. Thus, understanding the demands imposed by the CNRep is not difficult (9). The CNRep offers minimal opportunity for drawing on prior learned knowledge in performing the task because stimuli are not so familiar as words, and the task involves processing novel material as opposed to the Digit Span Test that relies heavily on prior knowledge of numbers (10). Indeed, pseudoword repetition ability has been considered an excellent marker for specific language impairment (10) and for phonological short-term memory deficits (11).

The CNRep was developed in accordance with the phonotactic rules of the English language. Since the stress, the pattern and the average number of syllables in Portuguese words are different, the original stimuli are not suitable for non-English speakers. It would be of value to Portuguese-speaking researchers of child memory and language development to have a Portuguese version of the CNRep. As far as we know, no previous attempt to produce such a version has been published, and therefore we developed a Portuguese version of the CNRep, which we have named Brazilian Children's Test of Pseudoword Repetition (BCPR), based on the original test criteria: minimal articulatory requirements, syllable dominance, and prosody and phonetic rules of the native language (8).

\section{Elaboration of BCPR stimuli}

Several steps were necessary to establish criteria for developing pseudowords and to identify crucial variables involved in the performance of the task.

Phonological structure. We took words normally heard on TV programs to analyze their phonological structure: length, spelling, syllabic construction, and prosody.

The next step was to select words heard by preschool children in recent children's songs or in traditional Brazilian children's rhymes and poetry. The examples below are presented in both languages to show the need for a Portuguese version of the test. Syllables stressed in Portuguese are underlined. Examples: casa/house, gato/cat, chuva/ 
rain, barata/cockroach, anel/ring, parede/ wall, encantado/bewitched, amarelo/yellow, felicidade/happiness. The dominant syllabic stress pattern in Portuguese words is strongweak for 2-syllable words (such as parte/portion), weak-strong-weak for 3-syllable words (cidade/city; escola/school), weak-weakstrong-weak for 4-syllable words (borboleta/ butterfly), and weak-weak-weak-strong-weak for 5-syllable words (analfabeto/illiterate).

Syllabic stress. To create stimuli with dominant syllable stress we excluded verbs, diphthongs, words with orthographic stress and words with $\mathrm{r}, \mathrm{m}, \mathrm{i}$ as the final letter because they have a different syllabic stress pattern. Double consonants, however, were accepted, as in ninho/nest, cachorro/dog.

Forty pseudowords divided into sets of 10 for each number of syllables (from two to five) were constructed according to these criteria and are listed in Appendix 1.

Utterance. The usual utterance of each pseudoword was evaluated by asking six adults (20-30 years of age) of both genders, all undergraduate students, to repeat the 40 pseudowords spoken by the experimenter and to read the same stimuli aloud. They made very few mistakes during pseudoword repetition, showing that the task is easy for young adults of university level. We noticed that usually they spontaneously diverted their gaze away from the experimenter's face, probably as a strategy to keep their attention on auditory reception of the information. However, when the pseudoword seemed to be very unfamiliar, they tried to lip-read. In the reading task, the adult subjects were quite uniform in prosody and intonation of the pseudowords, despite different regional accents. Phonological differences were not found; hence we considered that these stimuli followed the natural stress of syllables in the Portuguese language as spoken in Brazil.

Procedure. Five children aged 4-6 years of both genders were assessed for BCPR performance using the experimenter's live voice and a cassette player recording. Chil- dren used lip-reading more often than adults. The use of a cassette player to prevent lipreading was ruled out because, in spite of familiarity training before testing, we observed that the children typically smiled and talked while stimuli were being presented and engaged in playing with the cassette player as a toy. So we used a white sheet of paper as a screen to prevent lip-reading, a procedure used with young children (12) and with children with specific language impairment (10).

Articulation rate. One of the factors responsible for changes in span depends on articulation rate, since it reflects rehearsal (13). To consider this factor, we assessed the articulation rate for the BCPR stimuli.

The experimenter repeated each BCPR stimulus twice and the articulation time was measured with a stop watch. A repeated measure ANOVA was carried out to compare both measures and number of pseudoword syllables. The mean articulation rate in seconds according to stimulus length was: $94.1 \pm 6.2$ for 2 -syllable, $107.1 \pm 11.3$ for 3 syllable, $110.6 \pm 4.4$ for 4 -syllable and 129.4 \pm 15.6 for 5 -syllable stimuli.

There was a length effect $[\mathrm{F}(3,36)=12.0$; $\mathrm{P}<0.0001$ ] indicating more articulation time for long stimuli. There was no difference between the two measures $[\mathrm{F}(1,36)=1.46$; $\mathrm{P}$ $=0.23 \mathrm{]}$. The interaction between factors $[\mathrm{F}(3,36)=3.51 ; \mathrm{P}=0.024]$ revealed that the articulation time was longer for 5-syllable nonwords in the first measure than in the second measure.

Wordlikeness. Twenty-eight adults were asked to read and rate each pseudoword on an analogical scale ranging from $1 \mathrm{~mm}$ (very different from a real word) to $100 \mathrm{~mm}$ (very similar to a real word). They were instructed to rate the wordlikeness of each pseudoword with general Portuguese words and not the similarity between the pseudoword and a particular real word. The scale was divided into five blocks: 1-20 mm (very different), 21-40, 41-60, 61-80, and 81-100 mm (very 
similar). The mean rating for all nonwords was calculated, with a mean score of $59.54 \pm$ 6.14. The ratings ranged from 47.55 to 71.03. Therefore, no pseudowords were at the extremes of the scale. In other words, our stimuli were neither completely similar to nor completely different from real Portuguese words. This was due to the fact that Portuguese words have typically regular stress, and consequently Portuguese nonwords also tend to be regularly stressed.

When the ratings were divided into three categories according to the wordlikeness ranges: 47-55 $\mathrm{mm}$ (low), 56-63 mm (medium) and 64-71 mm (high), 10 pseudowords were rated as low (mean $=51.62 \pm 2.85), 20$ as medium $(59.59 \pm 2.51)$, and 10 as high $(67.34 \pm 2.18)$ (Appendix 1). Therefore, the number of items rated as low and high wordlikeness were counterbalanced, i.e., 10 in each condition. A wordlikeness effect was found $[\mathrm{F}(2,37)=96.33 ; \mathrm{P}<0.0001]$, with a progressive increase in the rates from the low to high similarity pseudowords. These categories were used in the first study to investigate wordlikeness.

After all of these steps were taken the test was applied in two studies. In the first one, with a small sample, we were mainly interested in the reliability of our version. The second study, with a larger sample, focused on the validity of the test.

Prior to testing, informed written consent for both experiments was obtained from the children's parents. It was explained to each child that the experiment could be discontinued at any time. The Ethics Committee of Universidade Federal de São Paulo approved the study.

\section{Test-retest reliability study}

Material and Methods

\section{Participants}

Participants were 29 Brazilian children
(17 boys and 12 girls) aged 4-7 years (mean age $=5.4 \pm 1.0)$. Inclusion criteria were standard vocabulary scores over 89 and Digit Span from the Stanford-Binet Intelligence Scale Revised (SBIS-R) (14) within the normal range. The means scores were $14.7 \pm 2.1$ (raw score; IQ = 92) for vocabulary, $4.3 \pm$ 0.8 for Digit Span forward (DSF), and $1.8 \pm$ 1.2 for Digit Span backward (DSB).

\section{Procedure}

Brazilian Children's Test of Pseudoword Repetition. Each child was told at the beginning of the test that he/she would hear some "funny made-up word" which he/ she should try to repeat aloud. The items were presented in a constant sequence to all subjects, spoken by the experimenter with the mouth hidden by a sheet of paper in order to prevent lip-reading. The child was allowed $3 \mathrm{~s}$ to make the attempt to repeat the word before being prompted by the experimenter.

The experimenter spoke the next pseudoword in the sequence after allowing one repetition attempt on the previous item. Each attempt was scored 0 if the experimenter judged that the child had produced a sound that differed from the target pseudoword by one or more phonemes, and 1 if the repetition was judged to be phonologically accurate. In cases in which it was apparent from the child's spontaneous speech that a specific phoneme was consistently misarticulated, credit was given for the consistent substitution. Incorrect responses were annotated on the answer sheet by the experimenter. The total number of pseudowords spoken correctly was calculated for each child.

The test was repeated after an interval of 7-15 days.

\section{Results and Discussion}

Table 1 presents the results of the reliability study. In general, 4- and 5-year-old children performed worse than older chil- 
dren. For all ages, more accuracy was observed for short pseudowords than for long ones. Children repeated high similarity pseudowords better than low similarity ones, and more errors occurred in medium similarity pseudowords.

The general reliability of the measure across the period was high $(\mathrm{r}=0.81 ; \mathrm{P}<$ $0.01)$. The coefficients were $\mathrm{r}=0.87(\mathrm{P}=$ $0.01)$ for the age of 4 years, $r=0.73(\mathrm{P}=$ $0.03)$ for 5 years, $r=0.91(\mathrm{P}<0.01)$ for 6 years, and $\mathrm{r}=0.86(\mathrm{P}=0.05)$ for 7 years. BCPR showed a similar reliability when compared to CNRep ( $\mathrm{r}=0.77$ for 5 years and $\mathrm{r}=$ 0.80 for 7 years by CNRep) (8).

To investigate the data further, a 4 (age groups/between subjects) x 2 (test-retest/within subjects) x 4 (number of syllables/within subjects) repeated measures ANOVA was carried out, followed by the Tukey post hoc test, with the level of significance set at $\mathrm{P} \leq 0.05$. There was a significant length effect $[F(3,75)=32.61$; $\mathrm{P}<0.0001$ ], with the accuracy of repetition declining with increasing number of syllables, indicating the greater difficulty of longer items compared to short items. The test-retest reliability and the length effect evident in both assessments indicated that BCPR satisfies the original criteria of the CNRep.
There was no significant age effect on test scores $[\mathrm{F}(3,25)=0.40 ; \mathrm{P}=0.75]$, possibly due to the small sample size. Also, no interaction between age and length was observed $[\mathrm{F}(9,75)=0.89 ; \mathrm{P}=0.53]$. Thus, we may assume that the relative item difficulty did not change within this age range.

Practice improved BCPR performance $[\mathrm{F}(1,25)=21.77 ; \mathrm{P}<0.0001]$. The number of correct repetitions in the retest (mean score $=$ $33.24 \pm 5.4$ ) was higher than in the first assessment (mean score $=30.20 \pm 3.8$ ). Possibly a learning effect occurred because the interval between the two assessments was short, reducing the difficulty of the test items. However, the relative difficulties of the test items depending on their length remained constant, as indicated by the lack of interaction between test-retest and length $[\mathrm{F}(3,75)=0.78$; $\mathrm{P}=0.50]$. All other interactions were nonsignificant: between age and test-retest $[\mathrm{F}(3,75)=0.81 ; \mathrm{P}=0.49]$ and age, length and test-retest $[\mathrm{F}(9,75)=0.94 ; \mathrm{P}=0.49]$.

Wordlikeness effect was investigated in a further analysis (see Table 1). A 4 (age groups) x 3 (wordlikeness categories; low, medium and high) repeated measures ANOVA was carried out. No age effect was observed $[\mathrm{F}(3,25)=0.73 ; \mathrm{P}=0.54]$. How-

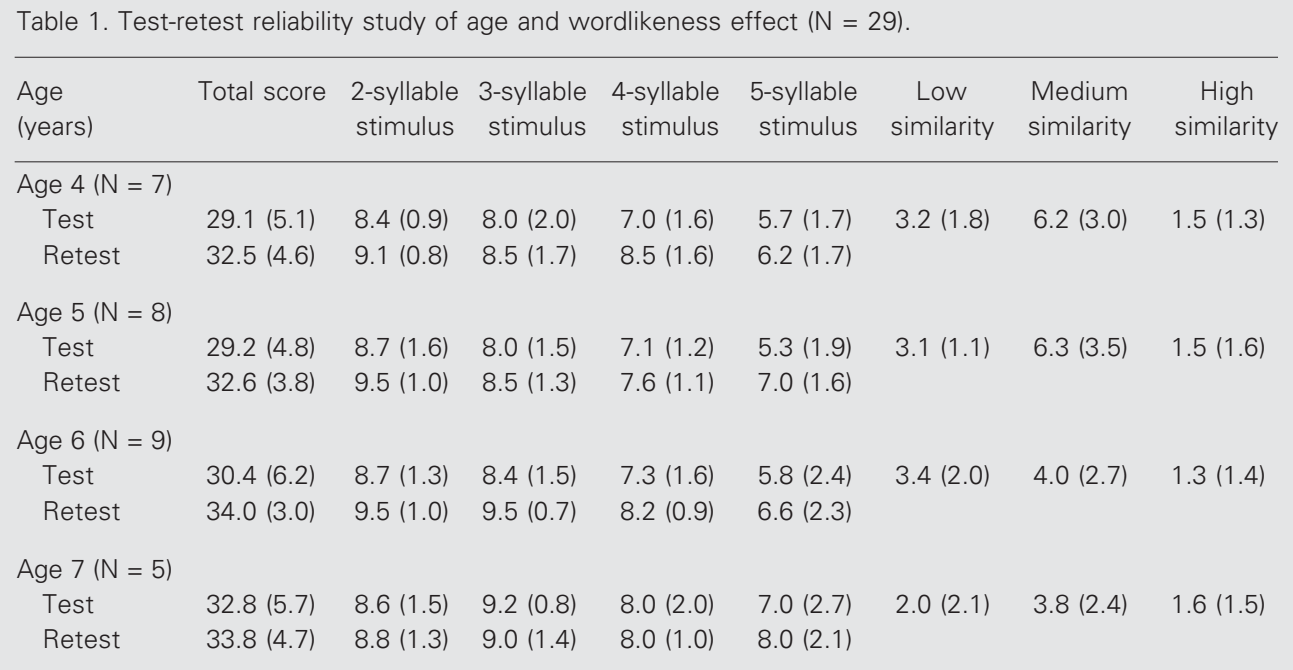

Data are reported as mean $( \pm$ SD) BCPR score (Brazilian Children's Test of Pseudoword Repetition). 
ever, a wordlikeness effect was found $[\mathrm{F}(2,50)=34.68 ; \mathrm{P}<0.0001]$, which revealed that children made more mistakes in pseudowords with medium wordlikeness than in pseudowords with high or low wordlikeness, and more mistakes in pseudowords with low than high wordlikeness. No interaction between age and wordlikeness was found $[F(6,50)=1.83 ; P=0.11]$. Therefore, wordlikeness differences occurred independently of age.

Our results repeated literature data (5) in that repetition accuracy was greater for pseudowords with high-rated $(64-71 \mathrm{~mm})$ wordlikeness than for pseudowords with lowrated $(47-55 \mathrm{~mm})$ wordlikeness. Second, medium-rated stimuli were more difficult than high- or low-rated stimuli. The length of pseudowords partially explains this difference since most pseudowords with high wordlikeness consisted of 2 syllables and were easier to repeat. On the other hand, most pseudowords with medium wordlikeness consisted of 4 or 5 syllables such as pseudowords with low wordlikeness. Perhaps this result was an artifact of the wordlikeness measurement procedure.

To better understand this question we calculated the number of errors for each nonword and divided the stimuli into two categories, i.e., easy and difficult repetition. This procedure might be considered an indirect measure of wordlikeness since in nonword repetition better performance is expected for nonwords similar to real words than for dissimilar stimuli (12). In other words, easy repetition corresponds to high wordlikeness, whereas difficult repetition corresponds to low wordlikeness. The frequency of errors ranged from 0 to 22 (mean score $=7.17 \pm 4.70$ ). The mean frequency of errors was equivalent to $24.7 \%$ of the responses and was used as cut off between categories. The stimuli were classified as 24 of easy repetition (mean score $=4.16 \pm 2.16$ ) and 16 of difficult repetition (mean score $=$ $11.68 \pm 3.75$ ). This procedure revealed that stimuli of difficult repetition ranged from 2 to 5 syllables ( 2 of 2 syllables, 2 of 3 syllables, 4 of 4 syllables, and 8 of 5 syllables). Then, ANOVA 2 (repetition; easy or difficult) $\mathrm{x} 4$ (length; 2 to 5 syllables) and number of errors was calculated. Both length $[\mathrm{F}(3,32)$ $=3.99 ; \mathrm{P}=0.015]$ and repetition $[\mathrm{F}(1,32)=$ 34.76; $\mathrm{P}<0.0001$ ] effects were demonstrable, but no interaction was found $[\mathrm{F}(3,32)=$ $0.37 ; \mathrm{P}=0.76]$. Therefore, better performance occurred for items classified as easy repetition regardless of stimulus length. Thus, easy repetition such as that observed for high wordlikeness stimuli is more related to longterm lexical knowledge and less sensitive to phonological memory constraints, while pseudowords of difficult repetition rely on phonological memory (5).

\section{Validity study}

The objective of this study was to obtain normative CNRep data by the validation of the BCPR for a larger sample of Portuguesespeaking subjects across different ages. As a criterion for validity we used Digit Span performance.

In the English normative study, significant correlations between DSF and CNRep by age were shown at age $4(\mathrm{r}=0.52)$, at age 5 $(r=0.66)$, and at age $8(r=0.44)$. Also, similar patterns for DSF and BCPR, such as age and schooling effects on performance, were used as validity criteria. In addition, DSB was assessed. This task taps more strongly than the forward version on the central executive component of working memory, since it requires both maintaining and manipulating (reversal of the order) the information. Correlations between BCPR and DSF are expected to be higher than between BCPR and DSB.

\section{Material and Methods}

\section{Participants}

The participants were 182 children, 89 
boys and 93 girls, aged 4 to 10 years. The children were raised in a rural region in the State of Minas Gerais $(\mathrm{N}=42)$, in an urban area from the same region of Minas Gerais $(\mathrm{N}=42)$, and in the city of São Paulo, SP $(\mathrm{N}=43)$.

The children were recruited in state supported schools from preschool to 4th grade. They were matched according to socioeconomic status (Brazilian Association of Marketing Research Institutes scale). Furthermore, the inclusion criterion was normal intellectual level as assessed by the RavenColored Matrices (15) for school children (percentiles over 50) or by the SBIS-R (14) for preschoolers (IQ over 89). The means were $23.23 \pm 4.2$ (raw score; percentile $=80$ ) for school children on the Raven scale and IQ was $97.9 \pm 6.0$ on the SBIS-R for preschoolers.

\section{Procedure}

The measures reported in this article represent a subset of a larger battery of tests administered to the children, such as visuospatial reasoning and visuospatial memory, which will be reported elsewhere. Children were always tested individually. The tests were administered in a single session and in randomized order. The following tests are those relevant to the present study:

$I$ - BCPR. It followed the standard procedure for the original test, as adopted in Study 1.

$I I$ - Digit Span - SBIS-R. This requires the forward (DSF) and backward (DSB) repetition of digit sequences of increasing length. In the DSF the child repeats the digits in the same order as the experimenter spoke them. In the DSB, the subject repeats the digits in reverse order. Two sequences of each extension were performed, the score being the maximum extension performed in forward and in backward order. The sequences and procedures were taken from the SBIS-R (14).

\section{Statistical analysis}

Three sets of analyses were performed. In the first, repeated measures ANOVA was carried out for both BCPR and Digit Span (DSF and DSB). In both cases, the betweensubject factors were 3 groups (urban from São Paulo, rural from Minas Gerais and urban from Minas Gerais), 7 ages (from 4 to 10 years) and 2 genders (male and female) or 5 schooling levels (from preschool to 4th grade), while the within-subjects factors were 4 numbers of syllables (from 2 to 5 ) for the BCPR and 2 orders (forward and backward) for the Digit Span.

In the second set, analyses of covariance (ANCOVA) using the same between and within factors were carried out - Digit Span as a covariant to the BCPR and vice versa - to investigate the factors that determine the main effects obtained. In the second and third sets of analyses the Tukey post hoc test was used with a significant alpha level of $\mathrm{P} \leq$ 0.05 . These last sets are shown in sequence for each variable.

In the third set, correlation and regression analyses between the two variables (BCPR and DSF or DSB) were carried out. For these analyses an alpha level of $\mathrm{P} \leq 0.01$ was adopted to minimize the effect of multiple comparisons.

Results

BCPR

Tables 2 and 3 present the results obtained with the BCPR by age and school grade. In general, the scores increased by age in both tests. DSF was performed better than DSB. Performance on BCPR decreased in long pseudowords for all ages. The scores were normally distributed (KolmogorovSmirnov test: $\mathrm{d}=0.08, \mathrm{P}=0.15)$.

Group and gender effects. A 2 (gender) $\mathrm{x}$ 3 (groups) ANOVA for the total score on BCPR was performed. Neither a gender ef- 
fect $[\mathrm{F}(1,121)=0.26 ; \mathrm{P}=0.87]$ nor a group effect $[\mathrm{F}(2,121)=1.88 ; \mathrm{P}=0.15]$ was found. Indeed, no interaction was observed $[F(2,121)=0.05 ; \mathrm{P}=0.94]$. Therefore, data from rural and urban regions were pooled; however, age and school grade factors were significant and the respective analyses are shown below.

Age effect. A 7 (age) x 4 (number of syllables) ANOVA was performed and an age effect was found: $[\mathrm{F}(6,175)=10.22 ; \mathrm{P}<$ 0.001 ]; 4- and 7-year-old children performed worse than 8-, 9- and 10-year-old children; 5- and 6-year-old children performed worse than 10-year-old children. A length effect $[F(3,525)=90.1 ; \mathrm{P}<0.0001]$ and an interaction between age and length $[\mathrm{F}(18,525)=$ 3.87 ; $\mathrm{P}<0.0001]$ were observed. Four-yearold children performed worse than 9- and 10-year-old children regarding 4-syllable items, and worse than 8-, 9- and 10-year-old children regarding 5-syllable items. However, when ANCOVA was calculated, with school grade as covariant, the age effect disappeared $[\mathrm{F}(6,173)=1.32 ; \mathrm{P}=0.24]$, whereas length effect $[\mathrm{F}(3,522)=90.8 ; \mathrm{P}<$ $0.0001]$ and age-length interaction $[\mathrm{F}(18,522)$ $=3.86 ; \mathrm{P}<0.0001]$ remained.

School grade effect. A 5 (school grade) $\mathrm{x}$ 4 (number of syllables) ANOVA was performed. The schooling effect $[\mathrm{F}(4,175)=$ $13,8 ; \mathrm{P}<0.001]$ was significant and preschool children performed worse than 2 nd, 3rd, and 4th grade children and 1st grade was worse than 4 th grade. A length effect $[F(3,528)=79.7 ; \mathrm{P}<0.0001]$ and an interaction between schooling and length were observed: $[\mathrm{F}(12,528)=5.59 ; \mathrm{P}<0.0001]$. Preschool children performed worse than 4 th grade children in repetition of 3-syllable words, worse than 3rd and 4th grades in repetition of 4-syllable words, and worse than 2nd to 4th grade children in repetition of 5-syllable words. ANCOVA, with age as covariant, showed that school grade $[\mathrm{F}(4,175)$ $=2.32 ; \mathrm{P}=0.05]$ and length $[\mathrm{F}(3,528)=$ $79.7 ; \mathrm{P}<0.0001]$ effects and their interaction $[\mathrm{F}(12,528)=5.59 ; \mathrm{P}<0.0001]$ persisted. Syllable number affects performance reflecting the item difficulty and length effect depends on schooling. Although an age effect was observed, this effect reflects the schooling level.

\section{Digit Span}

Tables 2 and 3 present the results obtained with the Digit Span test by age and school grade. Preschoolers scored less than other grades on Digit Span and BCPR, and higher on DSF than on DSB. Short pseudowords were repeated better than long ones by children of all grades.

Group and gender effects. First, a 2 (order) x 2 (gender) x 3 (group) repeated ANOVA for Digit Span was performed. No
Table 2. Validity study of Digit Span and BCPR by age $(N=182)$

\begin{tabular}{lcccccccc}
\hline $\begin{array}{l}\text { Age } \\
\text { (years) }\end{array}$ & $\mathrm{N}$ & $\begin{array}{c}\text { Digit Span } \\
\text { forward }\end{array}$ & $\begin{array}{c}\text { Digit Span } \\
\text { backward }\end{array}$ & $\begin{array}{c}\text { BCPR } \\
\text { total score }\end{array}$ & $\begin{array}{c}\text { BCPR } \\
\text { 2-syllable } \\
\text { stimulus }\end{array}$ & $\begin{array}{c}\text { BCPR } \\
\text { 3-syllable } \\
\text { stimulus }\end{array}$ & $\begin{array}{c}\text { BCPR } \\
\text { 4-syllable } \\
\text { stimulus }\end{array}$ & $\begin{array}{c}\text { BCPR } \\
\text { 5-syllable } \\
\text { stimulus }\end{array}$ \\
\hline Age 4 & 17 & $3.2(0.5)$ & $0.4(0.9)$ & $29.4(5.2)$ & $8.5(1.3)$ & $7.9(2.1)$ & $7.2(1.7)$ & $5.7(1.8)$ \\
Age 5 & 14 & $3.7(0.7)$ & $1.8(1.3)$ & $31.0(4.3)$ & $8.8(1.3)$ & $8.5(1.3)$ & $7.4(1.2)$ & $6.0(1.8)$ \\
Age 6 & 18 & $4.3(0.9)$ & $2.1(0.9)$ & $31.5(5.5)$ & $8.8(1.2)$ & $8.4(1.9)$ & $7.8(1.4)$ & $6.3(2.1)$ \\
Age 7 & 36 & $4.0(0.8)$ & $2.6(0.7)$ & $32.2(4.5)$ & $8.8(1.0)$ & $8.6(1.1)$ & $8.2(1.5)$ & $6.4(2.0)$ \\
Age 8 & 33 & $4.2(0.9)$ & $2.9(0.5)$ & $35.3(3.0)$ & $9.1(0.9)$ & $9.1(0.9)$ & $8.6(1.3)$ & $7.5(1.6)$ \\
Age 9 & 33 & $4.5(0.7)$ & $3.0(0.7)$ & $35.2(3.2)$ & $9.1(0.9)$ & $8.9(1.2)$ & $9.1(0.9)$ & $8.4(1.6)$ \\
Age 10 & 31 & $4.6(1.0)$ & $3.1(0.6)$ & $36.6(3.0)$ & $9.3(1.0)$ & $9.3(0.9)$ & $9.3(0.8)$ & $8.7(1.3)$
\end{tabular}

Data are reported as mean $( \pm$ SD) score for both Digit Span and BCPR (Brazilian Children's Test of Pseudoword Repetition). 
group effect was observed $[\mathrm{F}(2,124)=0.92$; $\mathrm{P}=0.40]$. DSF was better than DSB $[\mathrm{F}(1,124)=358.62 ; \mathrm{P}<0.0001]$ and an interaction was found $[\mathrm{F}(2,124)=6.10 ; \mathrm{P}=$ 0.003 ] that indicated that urban children from Minas Gerais performed the DSF better than rural children. No gender effect was observed $[F(1,180)=0.06 ; P=0.8]$. Children performed DSF better than DSB $[\mathrm{F}(1,180)=$ 460.8; $\mathrm{P}<0.0001]$ and no interaction was observed $[\mathrm{F}(1,180)=1.29 ; \mathrm{P}=0.25]$. Therefore, data from rural and urban regions were pooled. The analysis of age and school grade effects on Digit Span for the whole sample is given below.

Age effect. A 7 (age) x 2 (order) ANOVA was performed and revealed an age effect $[\mathrm{F}(6,175)=22.27 ; \mathrm{P}<0.0001] ;$ 4-year-old children performed worse than all other ages, 5-year-old performed worse than 8-, 9- and 10-year-old children, and 6- and 7-year-old children performed worse than 10-year-old children. Children performed DSF better than $\operatorname{DSB}[\mathrm{F}(1,175)=558.6 ; \mathrm{P}<0.0001]$, and an interaction was observed $[\mathrm{F}(6,175)=6.65$; $\mathrm{P}<0.0001]$. Four-year-old children performed forward digits worse than 6- to 10year-old children and for DSB, 4-year-old children performed worse than 5- to 10year-old children. These effects remained after ANCOVA was performed, with school grade as a covariant: age effect $[\mathrm{F}(6,173)=$ $8.22 ; \mathrm{P}<0.0001]$, order effect $[\mathrm{F}(1,174)=$ $553.51 ; \mathrm{P}<0.0001]$ and interaction $[\mathrm{F}(6,174)$
$=6.70 ; \mathrm{P}<0.0001]$.

School grade effect. A 5 (schooling) x 2 (order) ANOVA was performed. A significant schooling effect was obtained $[\mathrm{F}(4,176)$ $=20.4 ; \mathrm{P}<0.0001]$, with preschool children being impaired in relation to $2 \mathrm{nd}$, 3rd and 4th grades; 1 st grade children performed less well than 3rd and 4th grade children. Children performed DSF better than DSB $[\mathrm{F}(1,176)=406.3 ; \mathrm{P}<0.0001]$ and an interaction was found $[\mathrm{F}(4,176)=10.5 ; \mathrm{P}<$ 0.0001]. Preschool children performed DSF order worse than 3rd and 4th grade children. In DSB, preschool children performed worse than 1st to 4th grade children. However, when ANCOVA was performed, with age as covariant, the school grade effect was no longer observed $[\mathrm{F}(4,175)=0.63 ; \mathrm{P}<0.63]$. The main effect for order $[\mathrm{F}(1,176)=406.3$; $\mathrm{P}<0.0001]$ and interaction $[\mathrm{F}(1,176)=10.5$; $\mathrm{P}<0.0001]$ persisted. Thus, the results showed that Digit Span performance depends on age. Contrary to what was observed in BCPR performance, school grades reflect children's age.

\section{Correlations between BCPR and Digit Span}

The analyses were carried out by Pearson's product moment correlations. The correlations and respective degrees of freedom are summarized in Tables 4, 5 and 6 .

Correlation between variables. A correlation matrix was calculated, which revealed

\begin{tabular}{|c|c|c|c|c|c|c|c|c|}
\hline $\begin{array}{l}\text { School } \\
\text { grade }\end{array}$ & $N$ & $\begin{array}{l}\text { Digit Span } \\
\text { forward }\end{array}$ & $\begin{array}{l}\text { Digit Span } \\
\text { backward }\end{array}$ & $\begin{array}{c}\text { BCPR } \\
\text { total score }\end{array}$ & $\begin{array}{c}\text { BCPR } \\
\text { 2-syllable } \\
\text { stimulus }\end{array}$ & $\begin{array}{c}\text { BCPR } \\
\text { 3-syllable } \\
\text { stimulus }\end{array}$ & $\begin{array}{c}\text { BCPR } \\
\text { 4-syllable } \\
\text { stimulus }\end{array}$ & $\begin{array}{c}\text { BCPR } \\
\text { 5-syllable } \\
\text { stimulus }\end{array}$ \\
\hline Preschool & 54 & $3.8(0.8)$ & $1.5(1.2)$ & $30.8(5.1)$ & $8.7(1.2)$ & $8.3(1.7)$ & $7.6(1.5)$ & $6.1(2.0)$ \\
\hline 1st grade & 23 & $3.6(0.6)$ & $2.5(0.8)$ & $31.9(4.1)$ & $8.8(1.0)$ & $8.7(0.9)$ & $7.7(1.6)$ & $5.9(2.0)$ \\
\hline 2 nd grade & 36 & $4.2(0.9)$ & $3.1(0.6)$ & 34.4 (3.6) & $9.1(1.0)$ & $8.7(1.2)$ & $8.6(1.3)$ & $7.6(1.6)$ \\
\hline 3rd grade & 31 & $4.4(0.7)$ & $3.0(0.7)$ & 34.5 (3.8) & $8.9(1.0)$ & $9.1(1.1)$ & $9.0(0.9)$ & $7.9(1.8)$ \\
\hline 4 th grade & 36 & $4.7(0.9)$ & $3.0(0.6)$ & $36.7(2.6)$ & $9.4(0.7)$ & $9.2(0.9)$ & $9.3(0.7)$ & $8.8(1.2)$ \\
\hline
\end{tabular}

Data are reported as mean $( \pm$ SD) score for both Digit Span and BCPR (Brazilian Children's Test of Pseudoword Repetition). 
significant relationships between the measures used, except for that between 2-syllable pseudowords and DSB.

Correlation by age. A second correlation matrix was calculated, which revealed significant relationships between the measures used when the subjects were separated by age. BCPR performance was significantly related to DSF mainly in older children, while a correlation between BCPR and DSB was observed only in 5-year-old children.

Correlation by school grade. A third cor-

Table 4. Pearson correlations between BCPR and Digit Span by pseudoword length.

Digit Span forward Digit Span backward

BCPR score (total score) 2-syllable pseudowords

$0.50 *$

$0.19^{*}$

$0.30^{*}$

$0.43^{*}$

$0.50^{*}$

$0.43^{*}$

3-syllable pseudowords

4-syllable pseudowords

0.14

$0.22 *$

5-syllable pseudowords

$0.39 *$

$0.44^{*}$

$\mathrm{BCPR}=$ Brazilian Children's Test of Pseudoword Repetition. *Pearson coefficient (r); $P \leq 0.01, N=182$, d.f. $=180$.

Table 5. Pearson correlations between BCPR and Digit Span by age.

\begin{tabular}{lcc}
\hline Age (years) & Digit Span forward & Digit Span backward \\
\hline Age 4 & -0.10 & -0.22 \\
Age 5 & 0.39 & $0.68^{*}$ \\
Age 6 & 0.39 & 0.16 \\
Age 7 & $0.47^{*}$ & 0.18 \\
Age 8 & 0.39 & 0.04 \\
Age 9 & $0.56^{*}$ & 0.34 \\
Age 10 & $0.53^{*}$ & 0.23 \\
\hline
\end{tabular}

*Pearson coefficient $(r) ; P \leq 0.01, N=182$, d.f. $=180$.

Table 6. Pearson correlations between BCPR and Digit Span by school grade.

\begin{tabular}{lcc}
\hline School grade & Digit Span forward & Digit Span backward \\
\hline Preschool & $0.33^{*}$ & 0.25 \\
1st grade & 0.27 & 0.29 \\
2nd grade & $0.48^{*}$ & 0.20 \\
3rd grade & $0.68^{*}$ & 0.38 \\
4th grade & $0.35^{*}$ & 0.09 \\
\hline
\end{tabular}

${ }^{*}$ Pearson coefficient $(r) ; P \leq 0.01, N=182$, d.f. $=180$ relation matrix was calculated, which revealed significant relationships between measures when schooling factor was considered. BCPR performance was not significantly related to DSB. Correlation between DSF and BCPR was observed in all grades but the 1st.

Multiple regression. To describe a relationship between Digit Span tests and BCPR scores in more detail, a multiple regression was carried out with DSF and DSB as predictors for BCPR. In a stepwise regression analysis, DSF was entered first and explained $25.7 \%$ (R-square) of the variance in the BCPR score $[\mathrm{F}(1,180)=62.12 ; \mathrm{P}<0.01]$. DSB was entered second and accounted for a further $4.8 \%$ (increase of R-square from model 1 to model 2) of the variance in the BCPR score $[\mathrm{F}(1,179)=12.44 ; \mathrm{P}<0.01]$. The multiple regression model is summarized in Table 7.

Partial correlation. The partial correlation between DSF and BCPR (controlling for DSB) was only 0.38 instead of 0.50 while the partial correlation between DSB and BCPR (controlling for DSF) decreased from 0.43 to 0.25 , but both were still significant. This reduction was due to the fact that there was a correlation between DSF and DSB $(r=$ 0.47 ; $\mathrm{P}<0.001)$. Thus, DSF and DSB together explain $30.5 \%$ of the variance observed in BCPR with independent contributions. But high BCPR scores were associated with high scores for both predictors. Nevertheless, there remained almost $70 \%$ of variance in BCPR that could not be explained by the variance of the Digit Span scores. One can argue that this part of the variance reflects at least in part the higher sensitivity of BCPR for phonological memory, such as CNRep in comparison to other measures (5). The coefficients of the partial correlation are presented in Table 8 .

\section{Discussion}

The present data concern a Brazilian version of the CNRep, the BCPR assessed in 
182 Portuguese speakers between 4 and 10 years old from preschool to 4th grade in Brazilian state supported schools. Age, pseudoword length and schooling affected both BCPR and Digit Span, in agreement with the normative data analysis of the original CNRep (8). A further set of analyses was carried out to investigate the correlation between BCPR and Digit Span.

The BCPR scores of Portuguese speakers were higher than those obtained with English speakers. However, in both cases the mean score increased while the standard deviation decreased in older children. For instance, English children scored $18.7 \pm 6.0$ at age 4 and $32.8 \pm 4.3$ at age 9 in CNRep, less than Brazilians who scored $29.4 \pm 5.2$ at age 4 and $35.2 \pm 3.2$ at age 9. This quantitative difference might be the result of factors such as the differences in phonotactic and articulation rate between the two languages, and also by the live voice presentation that presumably facilitated the performance of Brazilian children.

In the present study, there was no age effect for 4- to 7-year-old children, and so the progression in scores by age in Portu- guese speakers seemed to be less linear than in English speakers. However, the sample size for 4- to 6-year-old Portuguese speakers was smaller than that of older ones compared to English speakers. This may be one reason for the lack of age effect among preschool children, and could also be the reason for the loss of age effect when schooling was considered separately as a covariant. However, evidence from neuroimaging and cognitive studies provides other explanations for the lack of age effect in younger children.

Neuroimaging studies have shown that the process of learning to read and write in infancy influences the functional architecture of the adult brain. For instance, illiterate adults experience greater difficulties than literate ones in tasks involving phonological processing, such as repetition of pseudowords (16).

A later study using a positron emission tomography scan and an auditory-verbal paradigm of word and pseudoword repetition applied to illiterate adults (17) showed different interactions between the languageinstrumental brain areas compared with literate individuals. According to this study,

Table 7. Summary of multiple regression analysis of the model.

\begin{tabular}{lccccccccc}
\hline Model & $R$ & $R^{2}$ & $\begin{array}{c}\text { Adjusted } \\
R^{2}\end{array}$ & $\begin{array}{c}\text { SD } \\
\text { estimate }\end{array}$ & $\begin{array}{c}R^{2} \\
\text { change }\end{array}$ & $\begin{array}{c}F \\
\text { change }\end{array}$ & d.f. 1 & d.f. 2 & $\begin{array}{c}\text { Significance } \\
\text { (F change) }\end{array}$ \\
\hline 1 & $0.507^{\mathrm{a}}$ & 0.257 & 0.252 & 3.9888 & 0.257 & 62.123 & 1 & 180 & 0.000 \\
2 & $0.552^{\mathrm{b}}$ & 0.305 & 0.287 & 3.8677 & 0.48 & 12.447 & 1 & 179 & 0.001 \\
\hline
\end{tabular}

aPredictors (constant) Digit Span forward. bPredictors (constant) Digit Span backward.

\begin{tabular}{|c|c|c|c|c|c|}
\hline Model & B & SD & Beta & $t$ & Significance \\
\hline 1 (constant) & 23.090 & 1.375 & & 16.797 & 0.000 \\
\hline DSF & 2.507 & 0.318 & 0.57 & 7.882 & 0.000 \\
\hline 2 (constant) & 22.931 & 1.334 & & 17.195 & 0.000 \\
\hline DSF & 1.923 & 0.350 & 0.388 & 5.491 & 0.000 \\
\hline DSB & 1.037 & 1.037 & 0.250 & 3.528 & 0.001 \\
\hline
\end{tabular}

Dependent variable: BCPR. DSF, DSB = Digit Span forward and backward, respectively. 
certain features of phonological processing are not acquired spontaneously but are modulated by learning a written language; more specifically, the acquisition of orthographic knowledge may modulate sublexical phonological processing and sublexical phonological awareness.

A close relationship between CNRep scores and reading tests was observed in British children aged 5 to 8 years, but not in those aged 4 , i.e., before the ability to read develops (8). Furthermore, the repetition of pseudowords was more efficient than Digit Span testing in differentiating reading ability between subjects. Indeed, a review of the literature about working memory development $(4,18)$ showed that the spontaneous use of active rehearsal does not appear in children until they achieve reading proficiency. Therefore, schooling demands might contribute to working memory skills.

Our observation of preschoolers seems to agree well with both neuroimaging and cognitive studies. The difficulty in nonword repetition declines with more effective reading and writing skills. These abilities are consolidated in the 3rd and 4th years of elementary school, particularly among Brazilian children who begin reading-writing classes around 7 years of age.

A recent study (19) compared twins with specific language impairment histories to twins from the general population in order to investigate genetic and environmental influences on the acquisition of literacy. Literacy problems and poor nonword repetition were frequent in specific language impairment subjects but not in the general population as a whole, in which literacy learning difficulties were significantly associated with environmental variables.

The length effect reflects the subvocal rehearsal component of phonological working memory. Repetition performance decreased as pseudowords increased from two to five syllables in length. This declining accuracy with increasing pseudoword length is consistent with the statement that pseudowords are temporarily retained in a time- or capacity-limited phonological memory system (6). The length effect has already been shown in young children (12), in normal children (8) and in preschool children (5). In the present report, a length effect was strongly evident in all subjects. For instance, differences related to age in 5-syllable pseudoword repetition occurred between preschool children and 8-, 9- and 10-year-old children, and differences related to schooling were observed between preschool and all grades. This is consistent with the idea that literacy improves performance since the correct analytical and perceptual processing of stimulus constituents, i.e., phonological segmentation, develops and consequently improves the phonological awareness $(7,20,21)$.

In the CNRep study (8), performance for 5-syllable pseudowords was consistently better than for 4-syllable pseudowords. The authors explained this by the prevalence of familiar morphological and phonological polysyllabic sequences (ex., altupatory, confratually, defermication, etc.) in 5-syllable pseudowords. This might have offset the decline in accuracy when maintaining increasingly lengthy phonological sequences in working memory.

This phenomenon was not observed in the present study. Brazilian children consistently performed 4-syllable better than 5syllable pseudowords. In our list of pseudowords, grammatical morphemes present in many words (e.g., panininha, belinidade, alvenioso) were used, but there was no repetition of the same suffix within each syllable length, and familiar grammatical morphemes were not prevalent in 5-syllable pseudowords. Another determinant of this difference probably is the well-established relationship between memory span and articulation time. A study (22) comparing subjects across a range of different languages found that performance on Digit Span and rate of digit reading were better when the language 
allowed quick reading of syllables and phonemes (e.g., in English) and worse in languages with longer vowel sounds (e.g., in Arabic). In our stimuli, 5-syllable pseudowords do really take more articulation time than 4-syllable ones and consequently requirements for storage accuracy in the phonological memory decline for 4-syllable pseudowords.

The Digit Span analysis showed that Digit Span scores are affected by the same factors as the BCPR score, providing evidence for the validity of the Portuguese version. However, some similarities with the BCPR and Digit Span were observed.

Age was a determinant of performance ability for Digit Span, an order effect was observed since in all sets of analyses, DSF was better performed than DSB. For DSF the differences occurred only from preschool to 3rd and 4th grades. On the other hand, in DSB better performance was evident in all grades compared to preschool children. Agerelated improvement in performance was seen regularly at each 12-month age band.

It has been shown that 6-year-old children use different acquisition strategies than older children and adults in DSF (18). Adults do actively use acquisition and retention strategies (e.g., grouping and rehearsal), older children can benefit from these strategies but do not adopt them spontaneously and younger children do not benefit due to a lack of maturity. Moreover, adults perform better at slower presentation rates ( 1 item per second) than at faster presentation (2.5 digits per second) whereas younger participants perform better at faster than at slower rates. Probably, there is echoic information for more items with faster presentation rates since there is less time for decay between items (23). Since in our study, according to the SBIS-R procedure, only slow presentation was used, we conclude that preschool children performed worse than older children since they had more difficulty in keeping the trace of memory and they may not spontaneously use memory strategies to avoid decay as older children do.

A pattern of strong association between pseudoword repetition and other phonological memory measures is accepted as evidence of the validity of pseudoword repetition as a test of phonological skills (8). In the present report, this association was confirmed by the significant correlation between BCPR total score and DSF $(r=0.50)$ and DSB $(r=$ 0.43 ). For subtest scores, both 4- and 5syllable stimuli showed the highest correlation with Digit Span in both orders. Previous research has shown a high correlation between multisyllable stimuli and total score for pseudoword repetition (6). This result suggests that 5-syllable pseudoword repetition score can be used as a predictor of Digit Span performance. Thus, in the absence of the Digit Span test, this score would be very useful for a rapid screening of this ability.

Correlation between BCPR and DSF was evident in older children ( 7,9 and 10 years of age) compared to preschoolers, while for the CNRep a correlation was found for 4-, 5and 8-year-old children. On the other hand, considering the schooling factor, correlation between BCPR and DSF was present from preschool to 4th grade, except for 1st grade. The highest correlation was found for 3rd grade $(r=0.68)$. Although the BCPR age correlation was progressive like in CNRep, the schooling factor showed almost a linear relationship between DSF and BCPR. Based on previous studies, we interpreted schooling to be more strongly related to this ability than age. Pseudoword repetition is a natural ability of the child that is present in younger children but accuracy of performance depends on phonological awareness, which is a consequence of perceptual and analytical processes supported by literacy (17).

It has been well established that DSB, besides being related to phonological memory, is also related to more complex processes, since it involves serial order. However, the correlation analysis did not support 
the idea that DSF and DSB are involved in different processes. Partial correlation analyses showed a persistent significant correlation between BCPR and DSF (when DSB was excluded) and between BCPR and DSB (when DSF was excluded). Also, there was a significant correlation between DSF and DSB $(\mathrm{r}=0.47)$. The present result is important because some studies do not note the reverse order contributions to phonological shortterm memory.

No correlation between BCPR and DSB related to schooling was found, although when the subjects were classified by age a correlation between BCPR and DSB was observed for 5-year-old children $(r=0.68)$. As shown in Table 4, children were unable to repeat back in reverse order two digits at age 4 and this ability started to develop at age 5 , increasing sharply up to 10 years. Developmental factors seem to be reflected in performance of tasks such as reverse order. For instance, the development of auditory brain areas extends up to the 4th year of life (24). The frontal cortex maturation is not completed before adolescence (25). Phonological memory seems to be established as early as about age 4, while executive processes develop over the subsequent years, and later both systems work independently but in association.
In summary, we developed and validated the BCPR - Brazilian Children's Test of Pseudoword Repetition, a phonological working memory task for Portuguese speakers aged 4 to 10 years, based on the English version, the CNRep (5). BCPR scores change with age and are influenced by schooling. Moreover, we obtained good reliability across ages and a correlation with Digit Span - the classical measure of phonological loop. We conclude that the BCPR is a useful tool to investigate speech production and working memory in Brazilian children and Portuguese speakers in general.

\section{Acknowledgments}

We especially thank the parents and children for their participation and acknowledge the cooperation of Escola Municipal de Educação Infantil São Paulo (EMEI-SP) and Fundação Julita, São Paulo, and Órgão Municipal de Educação de Brazópolis, Minas Gerais, Brazil. We also thank Teresa Candal for interviewing the children's parents, Claudia Mello for assessing part of the sample, Michael Ziessler for assistance with the statistical analyses and for reviewing an earlier draft of this paper, Susan Gathercole for helpful comments, and the anonymous referee for useful remarks.

Appendix 1 - Wordlikeness rating for the Brazilian Children's Test of Pseudoword Repetition (BCPR).

\begin{tabular}{|llll|}
\hline Low wordlikeness & \multicolumn{2}{c|}{ Medium wordlikeness } & High wordlikeness \\
\hline Porate & Renco & Envastado & Jama \\
Muralito & Pibo & Micharrinho & Fasta \\
Cocarelo & Serdelho & Limarado & Borca \\
Cormadura & Mantura & Belinidade & vana \\
Escurrama & Ampisco & Paripadura & muca \\
Apardicha & Talugo & Apapilado & lajo \\
Pergaleta & Barita & Incovilente & vesta \\
Alvenioso & Begina & Cabajucaba & riga \\
Melanitito & Magalo & Calentonina & Volinho \\
Novelitiva & Panininha & Rolinicista & Galvado \\
\hline
\end{tabular}




\section{References}

1. Baddeley AD \& Hitch GJ (1974). Working memory. In: Bower G (Editor), The Psychology of Learning and Motivation. Vol. 8. Academic Press, London, 47-90.

2. Baddeley AD (1986). Working Memory. Oxford University Press, Oxford, UK

3. Baddeley AD (1997). The role of memory in cognition: working memory. In: Baddeley AD (Editor), Human Memory: Theory and Practice. Psychology Press, Hove, UK, 49-69.

4. Baddeley AD, Gathercole SE \& Papagno C (1998). The phonological loop as a language learning device. Psychological Review, 105: 158-173.

5. Gathercole SE (1995). Is nonword repetition a test of phonological memory or long-term knowledge? It all depends on the nonwords. Memory and Cognition, 23: 83-94.

6. Gathercole SE, Willis CS, Baddeley AD \& Emslie H (1991). The influences of number of syllables and wordlikeness in children's repetition of nonwords. Applied Psycholinguistics, 12: 349-367.

7. Snowling M, Chiat S \& Hulme C (1991). Words, nonwords and phonological processes: Some comments on Gathercole, Willis, Emslie and Baddeley. Applied Psycholinguistics, 12: 369-373.

8. Gathercole SE, Willis CS, Baddeley AD \& Emslie H (1994). The children's test of nonword repetition: a test of phonological working memory. Memory, 2: 103-127.

9. Laws G (1998). The use of nonword repetition as a test of phonological memory in children with Down syndrome. Journal of Child Psychology and Psychiatry, 39: 1119-1130.

10. Bishop DVM, North T \& Donlan C (1996). Nonword repetition as a behavioural marker for inherited language impairment: Evidence from a twin study. Journal of Child Psychology and Psychiatry, 37: 3-27.

11. Gathercole SE (1998). The development of memory. Journal of Child Psychology and Psychiatry, 39: 3-27.

12. Adams A-M \& Gathercole SE (1995). Phonological working memory and speech production in preschool children. Journal of Speech and Hearing Research, 33: 210-219.

13. Cowan N, Wood NL, Wood PK, Keller TA, Nugent LD \& Keller CV (1998). Two separate verbal processing rates contributing to shortterm memory span. Journal of Experimental Psychology: General,
127: $141-160$

14. Thorndike RL, Hagen EP \& Sattler JM (1986). Technical Manual for the Fourth Edition Stanford-Binet Intelligence Scale (SBIS-R). The Riverside Publishing Company, Riverside, Chicago, IL, USA.

15. Angelini AL, Alves ICB, Custódio EM, Duarte VF \& Duarte JLM (1999). Manual de Matrizes Progressivas Coloridas de Raven Escala Especial. Centro Editor de Testes e Pesquisas em Psicologia, São Paulo, SP, Brazil.

16. Castro-Caldas A, Petersson KM, Reis A, Stone-Elander S \& Ingvar M (1998). The illiterate brain. Learning in childhood influences functional organization of adult brain. Brain, 121: 1053-1063.

17. Petersson KM, Reis A, Askelof S, Castro-Caldas A \& Ingvar $M$ (2000). Language processing modulated by literacy: a network analysis of verbal repetition in literate and illiterate subjects. Journal of Cognitive Neuroscience, 12: 364-382.

18. Gathercole SE \& Baddeley AD (1993). Working Memory and Language. Erlbaum, Hillsdale, NJ, USA.

19. Bishop DVM (2001). Genetic influences on language impairment and literacy problems in children: same or different? Journal of Child Psychology and Psychiatry, 42: 189-198.

20. Norrelgen F, Lacerda F \& Forssberg H (1999). Speech discrimination and phonological working memory in children with ADHD. Developmental Medicine and Child Neurology, 41: 335-339.

21. Metsala JL (1999). Young children's phonological awareness and nonword repetition as a function of vocabulary development. Journal of Educational Psychology, 91: 3-19.

22. Naveh-Benjamin \& Ayres TJ (1986). Digit span, reading rate and linguistic relativity. Quarterly Journal of Experimental Psychology, 38: 739-751.

23. Engle RW \& Marshall K (1983). Do developmental changes in Digit Span result from acquisition strategies? Journal of Experimental Child Psychology, 36: 429-436.

24. Kagan J (1985). The human infant. In: Rogers AM \& Scheirer CJ (Editors), The G. Stanley Hall Lecture Series. Vol. 5. American Psychological Association, Washington, DC.

25. Yakovelevi PI \& Lecours AR (1967). The myelogenetic cycles of regional maturation of the brain. In: Minkowski A (Editor), Regional Development of the Brain. Blackwell, Oxford, UK. 